\title{
Isobavachalcone inhibits the proliferation and invasion of tongue squamous cell carcinoma cells
}

\author{
YI SHI $^{1}$, WEI-ZHONG WU ${ }^{1}$, AN HUO ${ }^{1}$, WEI ZHOU ${ }^{1}$ and XIAO-HONG JIN ${ }^{2}$ \\ ${ }^{1}$ Department of Stomatology, Danyang People's Hospital of Jiangsu; ${ }^{2}$ Department of Oncology, \\ Yunyang People's Hospital of Danyang, Danyang, Jiangsu 212300, P.R. China
}

Received September 18, 2015; Accepted February 28, 2017

DOI: $10.3892 / \mathrm{ol} .2017 .6517$

\begin{abstract}
Isobavachalcone (2',4',4-trihydroxy-3'-[3'-methylbut-3'-ethyl] chalcone or IBC) exhibits anticancer activities in a number of types of cancer cell. However, its role in tongue squamous cell carcinoma (TSCC) cells remains unclear. The aim of the present study was to investigate the biological effect of IBC in TSCC Tca8113 cells. The function of IBC on Tca8113 cell apoptosis and apoptosis-associated signaling pathways was determined using an MTT assay, morphological staining, annexin V-propidium iodide (PI) staining and Western blot analysis. The effects of IBC on Tca8113 cell migration, invasion and relative protein expression were confirmed using wound healing analysis, Transwell invasion analysis and Western blot analysis, respectively. The results of the MTT assay and annexin V-PI staining indicated that IBC is able to significantly inhibit proliferation and induce apoptosis of Tca8113 cells in vitro. IBC treatment resulted in typical apoptotic morphology of nuclear fragmentation and apoptotic bodies in Tca8113 cells. Western blot analysis further demonstrated that IBC caused downregulation of the expression of B-cell lymphoma 2 (Bcl-2) protein, upregulation of the expression of Bcl-2-associated X protein (Bax), activation of caspases, and dephosphorylation of protein kinase B (Akt) and extracellular-signal-regulated kinase (ERK) proteins in a concentration- and time-dependent manner. The results of the present study suggest that IBC induces apoptosis in Tca8113 cells and that the induction may be associated with the activation of Bcl-2, Bax and caspase-3, and the inactivation of Akt and ERK. Furthermore, IBC inhibited migration and invasion of Tca8113 cells in vitro by downregulating matrix metalloproteinase (MMP)-2 and MMP-9 protein expression. The results of the present study indicate that IBC may be a potential anticancer drug for the treatment of TSCC.
\end{abstract}

Correspondence to: Ms. Xiao-Hong Jin, Department of Oncology, Yunyang People's Hospital of Danyang, 1 Yi Dian Yuan Road, Danyang, Jiangsu 212300, P.R. China

E-mail: jinxiaohong1986@163.com

Key words: isobavachalcone, tongue squamous cell carcinoma, proliferation, apoptosis, invasion, molecular mechanism

\section{Introduction}

Tongue squamous cell carcinoma (TSCC) is the most common malignant tumor in the oral and maxillofacial region (1). Compared with other oral malignant tumors, TSCC exhibits features of an increased degree of malignancy, more rapid proliferation and more marked invasion. Owing to the tongue's rich blood supply and frequent movement, TSCC often exhibits early cervical lymph node metastasis and increased transfer rates (2). Currently, the most effective treatment of TSCC is surgical resection. However, excessive surgical excision often affects oral function and postoperative quality of life in patients with TSCC (3). Following adjuvant treatment radiotherapy, chemotherapy or immunotherapy, the survival rate of patients with TSCC has markedly improved, but it remains unsatisfactory. Among the treatment options, chemotherapy has an important role in the comprehensive treatment of TSCC. As it exhibits side effects, including liver and kidney toxicity, inhibition of the hematopoietic system and damage to the body's immune system, its clinical application is limited to a certain extent (4). Therefore, the search for novel drugs with low toxicity and high efficacy is vital in the comprehensive treatment of TSCC. Traditional Chinese medicine primarily consists of the use of natural plants and minerals, which exhibit characteristics of low side effects and being rich in resources (5). Therefore, traditional Chinese medicine is a focus of research for antitumor drugs.

Psoralea corylifolia is an annual herb widely distributed in China. The fruit of $P$. corylifolia has been used in traditional Chinese medicine, exhibiting diuretic, analeptic and laxative effects. Pharmacology studies have demonstrated that $P$. corylifolia exhibits a number of biological activities including enhancement of immunity, antibacterial effects and treatment of vitiligo. Isobavachalcone (2',4',4-trihydroxy-3'-[3'-methylbut-3'-ethyl] chalcone or IBC) is a natural chalcone compound isolated from P. corylifolia. IBC exhibits anti-inflammatory, antifungal, antioxidant and antimicrobial pharmacological activities. Previously, it has been reported that IBC is able to promote tumor cell apoptosis without exhibiting toxic effects towards normal cells, including human neuroblastoma, ovarian cancer and gastric cancer cells (6-9). However, only a limited number of studies have been performed to investigate the effects of IBC on human TSCC. 
Furthermore, the antimicrobial activities of IBC may prevent infection during chemotherapy for patients with cancer (10). These results suggested that IBC as a newly discovered anticancer drug exhibited characteristics of low toxicity and high efficacy. IBC has the potential for clinical application to decrease the side effects of chemotherapy.

Therefore, the aim of the present study was to investigate the effect of various concentrations of IBC on human TSCC Tca8113 cells, including inhibition of cell proliferation, induction of apoptosis, and the ability to inhibit migration and invasion. In addition, changes in the expression of various proteins including the apoptosis-related protein kinase B (Akt), extracellular-signal-regulated kinase (ERK), Bcl-2-associated X protein (Bax), B-cell lymphoma 2 (Bcl-2) and caspase-3, and the invasion-related matrix metalloproteinase (MMP)-2 and MMP-9 were determined. These results provide a theoretical basis for the application of IBC in the clinical treatment of TSCC.

\section{Materials and methods}

Cell culture and treatment. Human TSCC Tca8113 cells were provided by Shanghai Institute of Biochemistry and Cell Biology (Shanghai, China). Tca8113 cells were cultured in RPMI-1640 medium (Gibco; Thermo Fisher Scientific, Inc., Waltham, MA, USA) supplemented with $10 \%$ fetal bovine serum (Gibco; Thermo Fisher Scientific, Inc.) and $100 \mathrm{U} / \mathrm{ml}$ penicllin/streptomycin (Nanjing KeyGen Biotech Co. Ltd., Nanjing, China), and maintained at $37^{\circ} \mathrm{C}$ in a humidified atmosphere containing $5 \% \mathrm{CO}_{2}$. The medium was changed three times/week. IBC was purchased from Shanghai Tauto Biotech Co., Ltd. (Shanghai, China). IBC was dissolved in dimethylsulfoxide (DMSO) as a 100-mM stock solution to give a final concentration of $0.1 \%$ in the culture medium.

MTT analysis. Tca8113 cells (5,000 cells/well) were seeded in 96-well cell culture plates and incubated overnight. Various concentrations $(10,20,40$ and $80 \mu \mathrm{M})$ of IBC were added, and cells were incubated for a further 24 or $48 \mathrm{~h}$. The control groups included a blank control, treated with RPMI-1640, and a negative control, treated with DMSO. The cells were incubated with $20 \mu 15 \mathrm{mg} / \mathrm{ml}$ MTT solution (Sigma-Aldrich; Merck KGaA, Darmstadt, Germany) for a further 4 h at $37^{\circ} \mathrm{C}$ in a humidified atmosphere containing $5 \% \mathrm{CO}_{2}$. Following lysis of the cells and dissolution of the formazan product in $150 \mu \mathrm{l}$ DMSO, the optical density (OD) was measured at $490 \mathrm{~nm}$ using a spectrophotometer (Bio-Rad Laboratories, Inc., Hercules, CA, USA). The proliferation inhibition rate was calculated using the following formula: Proliferation inhibition rate $=1$-(experimental sample OD-blank control sample OD)/(negative control sample OD-blank control sample OD) $\mathrm{x} 100 \%$. All experiments were performed in triplicate and mean values were plotted to create cell proliferation inhibition curves.

Morphological staining. Tca8113 cells were cultured in 6-well culture plates at a density of $5 \times 10^{5}$ cells/well. Tca8113 cells were treated with $40 \mu \mathrm{M}$ of IBC and incubated for $48 \mathrm{~h}$; control cells received no treatment. Following treatment, the cells were collected and centrifuged at $300 \mathrm{x} \mathrm{g}$ for $5 \mathrm{~min}$ at room temperature. The cell smears were stained with Wright-Giemsa solution (Sigma-Aldrich; Merck KGaA) for
$15 \mathrm{~min}$, and washed with running water. Alterations in cellular morphology were observed using light microscopy.

Annexin $V$ and propidium iodide (PI) staining. Tca8113 cells were cultured in 6-well culture plates at a density of $5 \times 10^{5}$ cells/well. Tca8113 cells were incubated for $48 \mathrm{~h}$ with various concentrations $(0,20$ and $40 \mu \mathrm{M})$ of IBC. The cells were collected, and centrifuged at $200 \mathrm{x}$ g for $5 \mathrm{~min}$ at room temperature. The cells were washed with PBS and suspended in binding buffer (Nanjing KeyGen Biotech Co. Ltd.). Cells were incubated with $5 \mu \mathrm{l}$ annexin $\mathrm{V}$-fluorescein isothiocyanate and $5 \mu \mathrm{l}$ PI (both Nanjing KeyGen Biotech Co. Ltd., Nanjing, China) for $15 \mathrm{~min}$ in the dark at room temperature. The percentage of apoptotic cells was evaluated using the BD FACSVerse flow cytometer (BD Biosciences, San Jose, CA, USA).

Wound healing analysis. Tca8113 cells were cultured in 6-well culture plates at a density of $5 \times 10^{5}$ cells/well, and incubated in RPMI-1640 medium supplemented with $10 \%$ fetal bovine serum and $100 \mathrm{U} / \mathrm{ml}$ penicillin/streptomycin. A central denuded wound was made in a confluent cell monolayer using a $200 \mu \mathrm{l}$ pipette tip. Subsequently, cells were cultured in serum-free RPMI-1640 containing 0,20 or $40 \mu \mathrm{M}$ IBC. Wound closure was observed, and images were captured under an inverted microscope 0 and $24 \mathrm{~h}$ after treatment. Relative cell migration was calculated using the formula: Relative cell migration $=($ mean distance of original wound-mean distance of $24 \mathrm{~h}$ wound)/mean distance of original wound $\mathrm{x} 100 \%$.

Transwell invasion analysis. Transwell invasion analysis was determined using a Transwell permeable support system (BD Biosciences, San Jose, CA, USA) containing 24-well Transwell chambers ( $8 \mu \mathrm{m}$ pore size polycarbonate membranes). The upper chamber was coated with $30 \mu 1$ Matrigel (BD Biosciences, San Jose, CA, USA). Tca8113 cells and various concentrations $(0,20$ and $40 \mu \mathrm{M})$ of IBC were seeded into the upper chambers at densities of $5 \times 10^{4}$ cells/well in serum-free RPMI-1640. RPMI-1640 containing $600 \mu 110 \%$ fetal bovine serum was added to the lower chamber as a chemoattractant. Following incubation at $37^{\circ} \mathrm{C}$ for $24 \mathrm{~h}$, the cells on the lower surface of the polycarbonate membranes were fixed with $4 \%$ formaldehyde and stained with $0.5 \%$ crystal violet. The invading cells were counted visually in five random fields at x400 magnification using light microscopy.

Western blot analysis. Tca8113 cells were cultured in 6-well culture plates at a density of $5 \times 10^{5}$ cells/well. Following treatment with IBC $(0,20$ and $40 \mu \mathrm{M})$ for $48 \mathrm{~h}$, Tca8113 cells were washed with ice-cold PBS and protein was extracted with lysis buffer containing $50 \mathrm{mmol} / \mathrm{l}$ Tris- $\mathrm{HCl}, \mathrm{pH} 7.4,150 \mathrm{mmol} / \mathrm{l}$ $\mathrm{NaCl}, 1 \%$ Triton $\mathrm{X}-100,1 \mathrm{mmol} / 1$ EDTA, 0.1\% SDS and $2 \mathrm{mmol} / \mathrm{l} \mathrm{PMSF}$. The protein concentrations in the supernatant were estimated using a BCA Protein Assay kit (Beyotime Institute of Biotechnology, Haimen, Jiangsu, China) using bovine serum albumin as a standard. Samples of $20 \mu 1$ protein were separated by SDS-PAGE (12\% gel) and then transferred onto polyvinylidene fluoride membranes. Following incubation for $1 \mathrm{~h}$ in blocking buffer (5\% non-fat dry milk in PBS containing Tween-20), the membranes were incubated with the primary antibodies overnight at $4^{\circ} \mathrm{C}$. Primary antibodies 


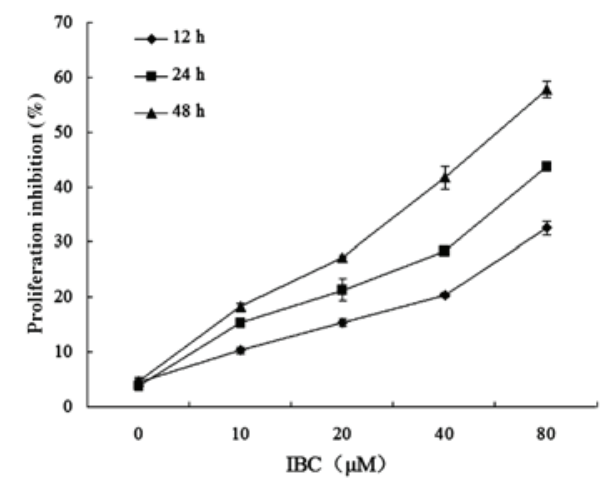

Figure 1. IBC inhibits Tca 8113 cell proliferation. TSCC cells were incubated for 12,24 or $48 \mathrm{~h}$ with $0,10,20,40$ or $80 \mu \mathrm{M}$ IBC. Results are expressed as the mean \pm standard deviation of three independent experiments. IBC, isobavachalcone; TSCC, tongue squamous cell carcinoma.

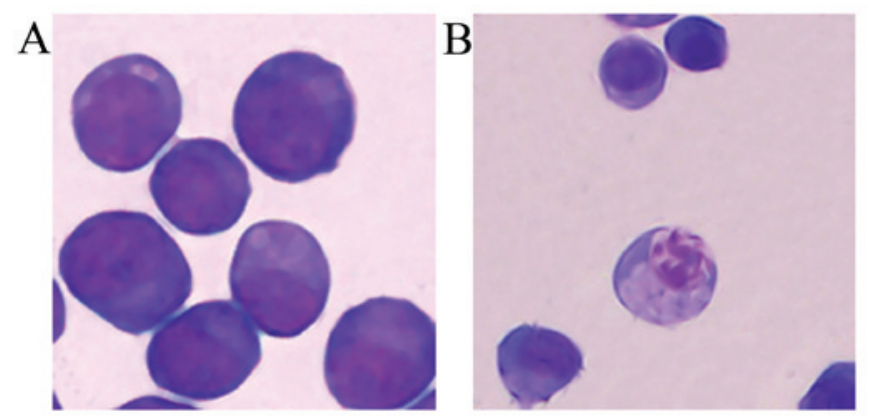

Figure 2. Analysis of apoptotic morphology in Tca8113 cells (magnification, $\mathrm{x} 400$ ). At $48 \mathrm{~h}$ after incubation with (A) 0 or (B) $40 \mu \mathrm{M} \mathrm{IBC,} \mathrm{Tca8113} \mathrm{cells}$ were detected with Wright-Giemsa staining. IBC, isobavachalcone.

were as follows: Akt (dilution, 1:200; cat. no., sc-8312); p-Akt (dilution, 1:200; cat. no., sc-135650); ERK (dilution, 1:200; cat. no., sc-94); p-ERK (dilution, 1:200; cat. no., sc-16982); Bax (dilution, 1:300; cat. no., sc-493); Bcl-2 (dilution, 1:300; cat. no., sc-492); caspase-3 (dilution, 1:200; cat. no., sc-7184); MMP-2 (dilution, 1:200; cat. no., sc-10736); MMP-9 (dilution, 1:200; cat. no., sc-10737); $\beta$-actin (dilution, 1:1,000; cat. no., sc-1616); all antibodies were from Santa Cruz Biotechnology (Dallas, TX, USA). The membranes were then treated with horseradish peroxidase-conjugated secondary antibodies, including chicken anti-mouse (cat. no., sc-2954) and chicken anti-rabbit (cat. no., sc-2955; both dilution, 1:800; Santa Cruz Biotechnology. Inc.) and chemiluminescence substrate (ECL plus, Beyotime Institute of Biotechnology, Haimen, Jiangsu, China). The digital images were captured and analyzed densitometrically using ImageJ 2X software (National Institutes of Health, Bethesda, MD, USA).

Statistical analysis. Statistical analysis was carried out using SPSS for Windows (version 16.0; SPSS, Inc., Chicago, IL, USA). Results were expressed as the mean \pm standard deviation. Multi-group comparisons of the means were carried out by one-way analysis of variance test, and the post hoc analysis was performed using the Student-Newman-Keuls test. $\mathrm{P}<0.05$ was considered to indicate a statistically significant difference. Three independent experiments were performed for all assays.
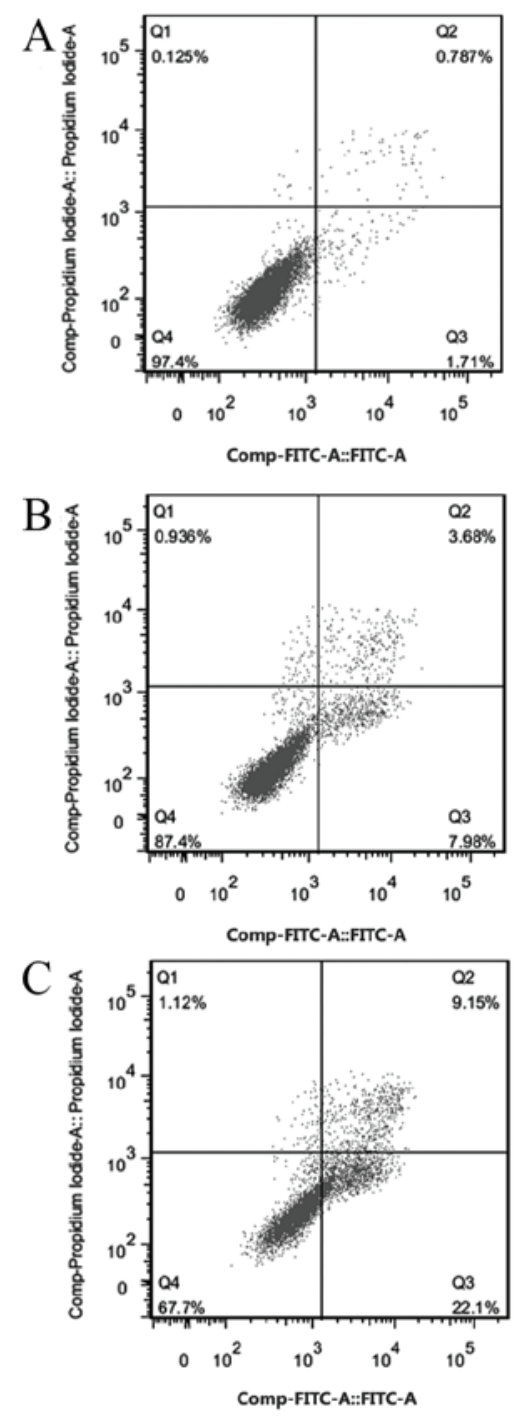

Figure 3. Analysis of annexin V-FITC/propidium iodide staining to assess the pro-apoptotic ability of IBC in Tca8113 cells. At $48 \mathrm{~h}$ after incubation with (A) 0 , (B) 20 or (C) $40 \mu \mathrm{M}$ IBC, Tca8113 cells were detected using flow cytometry. FITC, fluorescein isothiocyanate; IBC, isobavachalcone; Q, quadrant.

\section{Results}

IBC inhibits the proliferation of Tca8113 cells. Tca8113 cells were treated with between 0 and $80 \mu \mathrm{M} \mathrm{IBC}$ for 12,24 and $48 \mathrm{~h}$. An MTT assay demonstrated that the half-maximal inhibitory concentrations of IBC at 12,24 and $48 \mathrm{~h}$ were $285.13 \pm 8.97$, $132.40 \pm 7.76$ and $58.56 \pm 5.93 \mu \mathrm{M}$, respectively ( $\mathrm{P}<0.05$; Fig. 1).

IBC induces the apoptotic morphology of Tca8113 cells. Tca8113 cells were treated with $40 \mu \mathrm{M}$ IBC for $48 \mathrm{~h}$ to observe the alterations in cell morphology. IBC treatment resulted in cell shrinkage and nuclear DNA fragmentation in Tca 8113 cells (Fig. 2), suggesting that IBC induced apoptosis in Tca8113 cells.

IBC induces the apoptosis of Tca8113 cells. Tca8113 cells were cultured in 20 and $40 \mu \mathrm{M}$ IBC for $48 \mathrm{~h}$. The pro-apoptotic rates were $8.21 \pm 2.32$ and $22.45 \pm 1.18 \%$, respectively, which were significantly increased compared with the untreated control $(1.69 \pm 0.65 \%)(\mathrm{P}<0.05$; Fig. 3). These results suggested 

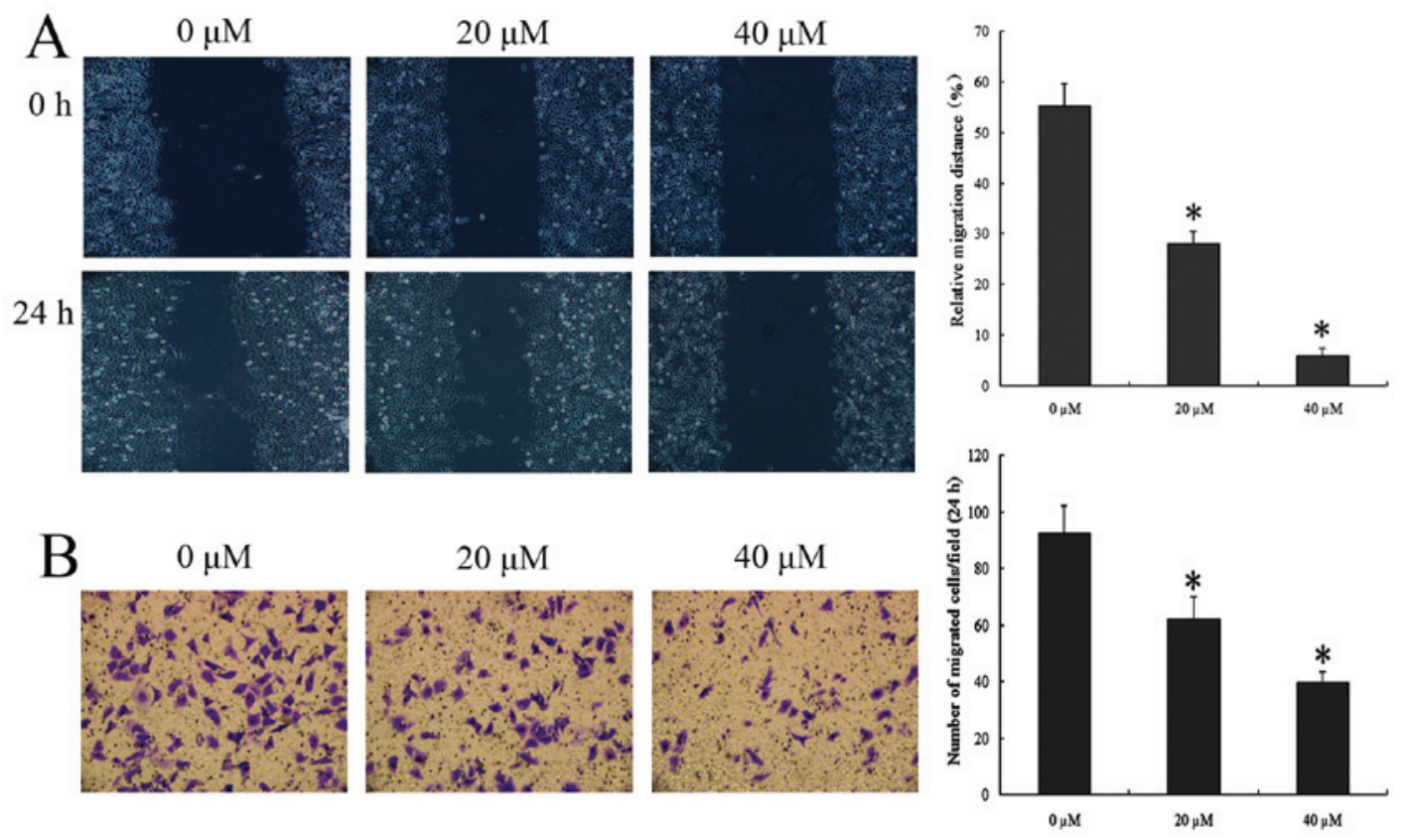

Figure 4. IBC decreases the migration and invasion of Tca8113 cells. (A) Wound healing analysis of the effect of IBC on the migration of Tca8113 cells. (B) Transwell invasion analysis of the effect of IBC on the invasion of Tca8113 cells. At $24 \mathrm{~h}$ after incubation with 0,20 or $40 \mu \mathrm{M}$ IBC, Tca8113 cells were observed using a light microscope (magnification, $\mathrm{x} 200$ ). Results are expressed as the mean \pm standard deviation of three independent experiments. ${ }^{*} \mathrm{P}<0.05$ vs. untreated control. IBC, isobavachalcone.

that IBC treatment induced apoptosis in Tca8113 cells. The cell apoptosis rate was positively associated with drug concentration.

IBC inhibits the migration and invasion of Tca8113 cells. To determine whether IBC treatment has an effect on wound repair, wound healing was compared between Tca8113 cells with and without IBC. To eliminate the effect of cell apoptosis on the results, an IBC treatment time of $24 \mathrm{~h}$ was selected rather than $48 \mathrm{~h}$. The results of the wound healing and Transwell invasion analysis demonstrated the effect of IBC on the migration and invasion of Tca8113 cells respectively. The migration and invasion ability of 20 and $40 \mu \mathrm{M}$ IBC-treated Tca8113 cells were significantly decreased compared with the untreated control $(\mathrm{P}<0.05$; Fig. 4). The results indicated that IBC caused marked decreases in cell migration and invasion, indicating that IBC serves a vital role in cell migration.

Effect of IBC on the expression of Tca8113 cell apoptosis-related proteins. IBC treatment at 20 and $40 \mu \mathrm{M} \mathrm{IBC}$ resulted primarily in significant downregulation of the anti-apoptotic protein Bcl-2, and, in contrast, significant upregulation of the apoptotic protein $\mathrm{Bax}(\mathrm{P}<0.05$; Fig. 5). Members of the caspase protein family are known to induce apoptosis in cancer cells. As presented in Fig. 5, IBC treatment with 20 and $40 \mu \mathrm{M}$ IBC increased caspase-3 activity in Tca8113 cells. These results suggested that IBC treatment led to a concentration-dependent decrease in Bcl-2 expression, and a concentration-dependent increase in Bax and caspase-3 expression in Tca8113 cells.

A number of protein kinases (including Akt or ERK) are involved in cell proliferation and transformation. Therefore, the effect of IBC on activities of Akt and ERK in Tca8113 cells was investigated. It was identified that 20 and $40 \mu \mathrm{M}$ IBC treatment for $48 \mathrm{~h}$ significantly decreased the protein levels of phospho (p)-Akt and p-ERK compared with the untreated control in Tca8113 cells. In order to observe the effect of IBC on protein expression in Tca8113 cells following various incubation times, Tca8113 cells were cultured in $40 \mu \mathrm{M}$ IBC for 12,24 and $48 \mathrm{~h}$. It was identified that IBC treatment for $48 \mathrm{~h}$ significantly decreased the protein expression levels of p-Akt and p-ERK compared with the untreated control in Tca8113 cells $(\mathrm{P}<0.05$; Fig. 6). Total Akt and ERK levels were not affected by the concentration or duration of IBC treatment. These results suggested the ability of IBC to inactivate Akt and ERK in Tca8113 cells.

Effect of IBC on the expression of Tca8113 cell invasionrelated proteins. In order to identify the effect of IBC on the expression of Tca8113 cell invasion-related proteins, Tca8113 cells were treated with 20 and $40 \mu \mathrm{M}$ IBC for $48 \mathrm{~h}$ to observe the protein expression of MMP-2 and MMP-9 by Western blot analysis. It was identified that IBC significantly decreased MMP-2 and MMP-9 protein expression in Tca8113 cells compared with the control $(\mathrm{P}<0.05$; Fig. 7$)$. These results indicated that IBC may regulate the protein expression of MMP-2 and MMP-9 at the transcriptional level in TSCC cells.

\section{Discussion}

Malignant tumors are one of the most serious threats to human health. In comparison with normal cells, malignant tumor cells are able to divide and proliferate endlessly. Malignant tumor cells are able to damage the body's histological 

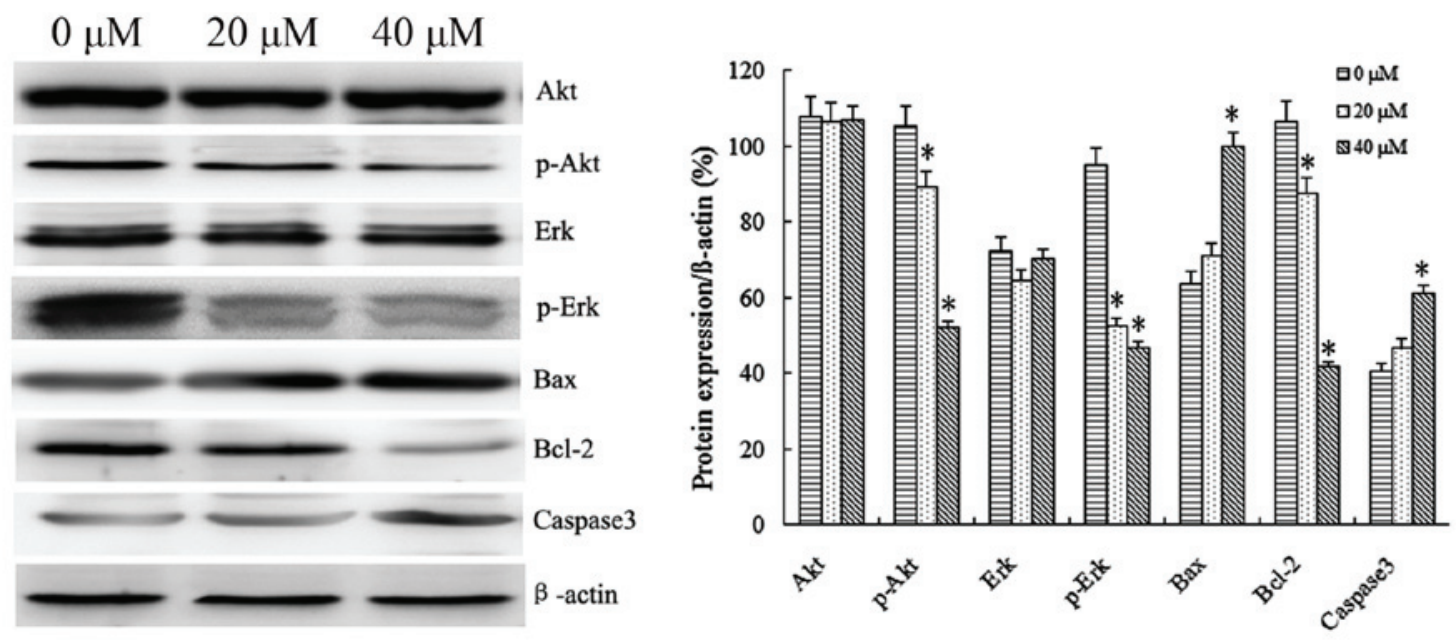

Figure 5. IBC regulates the protein expression of Akt, p-Akt, ERK, p-ERK, Bax, Bcl-2 and caspase-3 in Tca8113 cells in a concentration-dependent manner. At $48 \mathrm{~h}$ after incubation with 0,20 or $40 \mu \mathrm{M}$ IBC, the expression levels of all proteins were analyzed by western blotting. $\beta$-actin served as a loading control. Results are expressed as the mean \pm standard deviation of three independent experiments. "P<0.05 vs. control. IBC, isobavachalcone; Akt, protein kinase B; $\mathrm{p}$, phospho; ERK, extracellular-signal-regulated kinase; Bax, Bcl-2-associated X protein; Bcl-2, B-cell lymphoma 2.
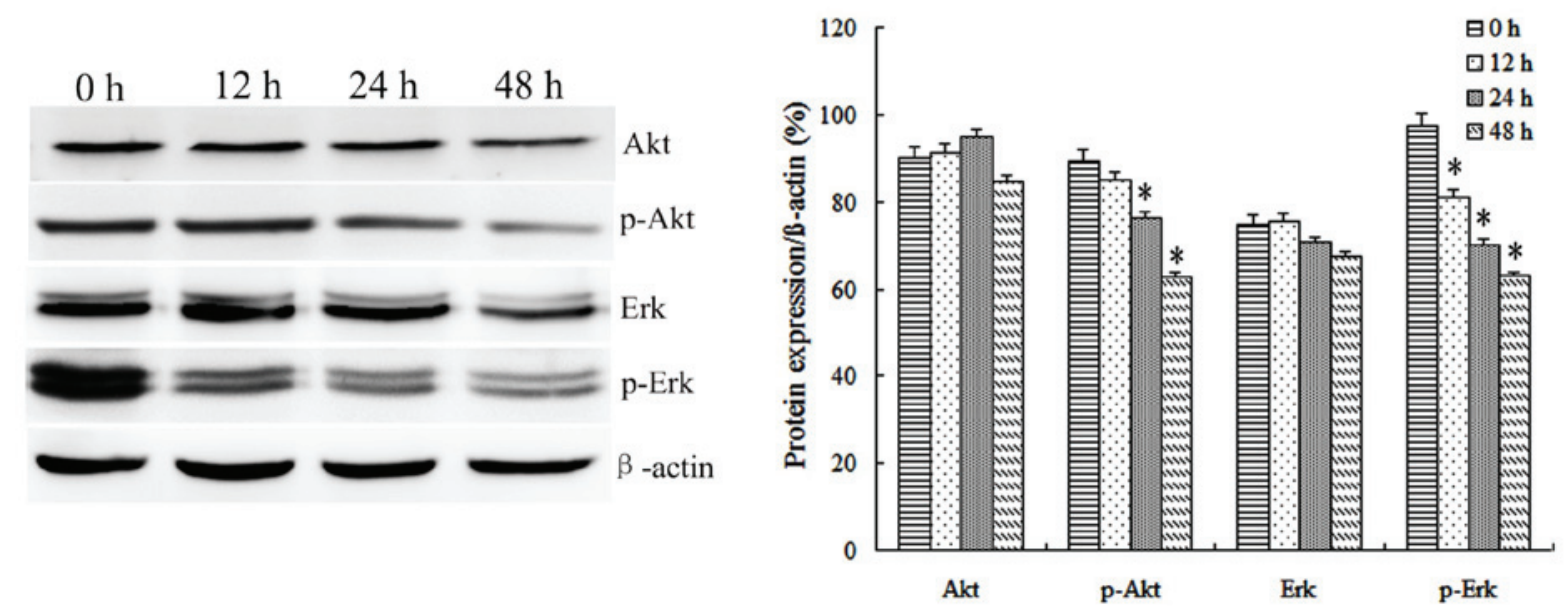

Figure 6. IBC regulates the protein expression of Akt, p-Akt, ERK and p-ERK in Tca8113 cells in a time-dependent manner. At $0,12,24$ and $48 \mathrm{~h}$ after incubation with $40 \mu \mathrm{M} \mathrm{IBC}$, the expression level of Akt and ERK proteins was analyzed by western blotting. $\beta$-actin served as a loading control. Results are expressed as the mean \pm standard deviation of three independent experiments. " $\mathrm{P}<0.05$ vs. control. IBC, isobavachalcone; Akt, protein kinase B; $p$, phospho; ERK, extracellular-signal-regulated kinase.
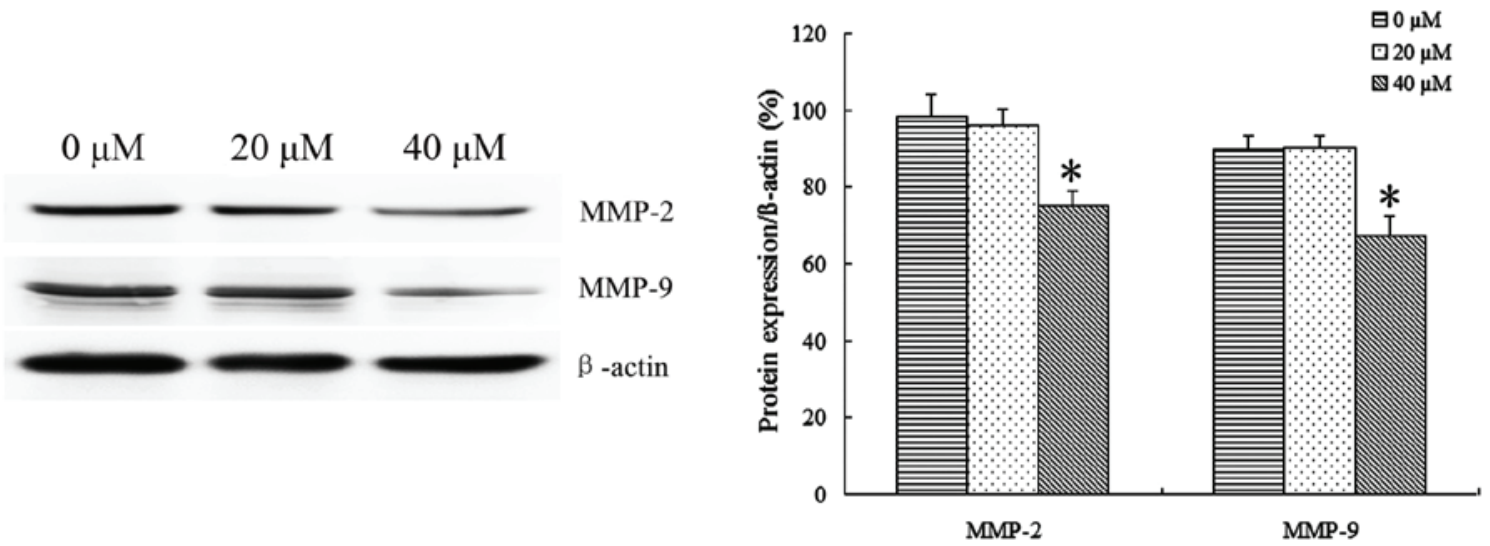

Figure 7. IBC regulates the protein expression of MMP-2 and MMP-9 in Tca8113 cells. At $48 \mathrm{~h}$ after incubation with 0,20 or $40 \mu \mathrm{M}$ IBC, the expression level of MMP-2 and MMP-9 proteins was analyzed by western blotting. $\beta$-actin served as a loading control. Results are expressed as mean \pm standard deviation of three independent experiments. $\mathrm{P}<0.05$ vs. control. IBC, isobavachalcone; MMP, matrix metalloproteinase. 
barrier by various means, invade normal tissue, metastasize, and spread via the bloodstream and lymphatic system (11). Current clinical treatments for TSCC are primarily surgery, radiotherapy, chemotherapy and immunotherapy. Surgery, which is the preferred method of TSCC treatment, is limited by tumor invasion, metastasis and a number of other factors. Chemotherapy, owing to its poor specificity, would also inevitably kill normal tissue cells and destroy the human immune system. The clinical application of chemotherapy drugs has become a 'double-edged sword'. Seeking novel ways to treat TSCC and the development of effective antitumor drugs has become important in modern medicine, particularly in the field of clinical medicine.

$P$. corylifolia is a legume member of the angiosperms. It has been demonstrated previously that a variety of active ingredients extracted from $P$. corylifolia are able to serve a antitumor role through various mechanisms (12). IBC, an active ingredient from $P$. corylifolia, was initially extracted by Bhalla et al (13) in 1968. It has been demonstrated that IBC exhibited antibacterial, antifungal, anti-inflammatory, antimicrobial and antitumor activities (14). In the development of a novel anticancer drug with low toxicity and cost, IBC is a promising candidate for clinical application. Previous in vitro experiments confirmed that, without damaging normal tissue cells, IBC inhibited the proliferation and induced apoptosis in a variety of tumor cells including OVCAR-8, PC3, A549 and MCF-7 cells (6-9). However, little is known about the association between IBC and human TSCC Tca8113 cells.

In the present study, the effect of IBC on cell proliferation and apoptosis of TSCC Tca8113 cells in vitro was determined using an MTT assay, Wright-Giemsa staining and flow cytometric analysis. Using an MTT assay, IBC demonstrated significant inhibition of cell proliferation in a marked concentration- and time-dependent manner. Tca8113 cells treated with $40 \mu \mathrm{M}$ IBC for $48 \mathrm{~h}$ demonstrated typical apoptotic morphology of nuclear fragmentation and apoptotic bodies. Furthermore, flow cytometric analysis identified pro-apoptotic rates of $8.21 \pm 2.32$ and $22.45 \pm 1.18 \%$ for Tca8113 cells treated with 20 and $40 \mu \mathrm{M}$ IBC for $48 \mathrm{~h}$, respectively, which were significantly increased compared with the untreated control. These results indicated that IBC inhibited the proliferation of Tca8113 cells and induced apoptosis in a concentration- and time-dependent manner.

The primary characteristics of malignant tumors is that tumor cells exhibit unrestricted proliferation and are able to circumvent apoptosis. Members of the Bcl-2 family encode anti-apoptotic proteins and pro-apoptotic proteins (15). Bcl-2 and Bax are, respectively, important anti-apoptotic and pro-apoptotic genes in the Bcl-2 gene family. The expression of $\mathrm{Bcl}-2$ protein is generally low in normal cells, but abnormally increased in tumor cells. The prognoses of cancer patients are markedly associated with $\mathrm{Bcl}-2$ protein expression (16). Zhang et al (17) observed the expression of $\mathrm{Bcl}-2$ protein in TSCC tissues using immunohistochemistry. They identified that, compared with the adjacent tissues, Bcl-2 protein expression was significantly increased in TSCC tissues. Increased expression of Bcl-2 was also markedly associated with platinum drug resistance in TSCC cells. Overexpression of Bcl-2 protein may inhibit apoptosis induced by growth factor deficiency, and caused by chemotherapy and radiotherapy (18).
Therefore, it hypothesized that there is an association between expression levels of $\mathrm{Bcl}-2$ protein in TSCC and tumor cell apoptosis. Decreasing the level of Bcl-2 protein expression may improve the drug resistance and low radiosensitivity of tumor cells, thereby enhancing the curative effect of chemotherapy and radiotherapy for patients with tongue cancer, and increasing the range of options for clinical treatment of TSCC. In the present study, using western blot analysis, the expression of Bcl-2 and Bax protein was observed in Tca8113 cells cultured with various concentrations of IBC for $48 \mathrm{~h}$. The results demonstrated that $\mathrm{Bcl}-2$ protein expression was significantly decreased in association with an increase in the concentration of IBC. However, Bax protein expression was significantly increased in association with an increase in IBC concentration.

The serine/threonine protein kinase Akt, an important target of phosphoinositide 3-kinase/Akt signal transduction pathway, may exhibit abnormal activation in a variety of malignant tumors including gastric, cervical and tongue cancer. Akt is able to phosphorylate and regulate a number of proteins which were associated with cell metabolism, apoptosis, proliferation and differentiation, thereby inhibiting the apoptosis of tumor cells and promoting the proliferation of tumor cells. Akt protein expression may be regulated to alter the balance between cell proliferation and apoptosis, to achieve the purpose of inhibiting tumor growth (19). In addition, p-Akt may phosphorylate Bcl-2-associated death promotor protein to inhibit its ability to bind to the B-cell lymphoma extra large protein, restoring the apoptosis resistance of Bcl-2 protein (20). ERK is a special type of cell signaling protein, whose function is to conduct the signal from the cell surface to the nucleus (21). Abnormal activation of the ERK signaling pathway has been demonstrated in a number of types of tumor cell, including TSCC cells, which has marked importance for the development of tumors. Our previous study demonstrated that IBC dose-dependently inhibited the activation of the Akt and ERK signaling pathways in gastric carcinoma MGC803 cells (22). These results are consistent with those of the present study that, under various concentrations or with various incubation times, IBC inhibited p-Akt and p-ERK protein expression. These results indicated that IBC may inhibit the Akt and ERK signaling pathways to promote the apoptosis of TSCC.

The present study observed the effects of IBC on Tca8113 cell migratory ability using wound healing analysis. It was identified that in association with the increase in drug concentration, the migratory ability of Tca8113 cells gradually increased. However, tumor invasion and metastasis is a complex process. Tumor cells metastasize following the degradation of extracellular matrix (ECM) and entry into the blood circulation or lymphatic system. In the present study, the effects of IBC on the Tca8113 cell invasive ability was observed using Transwell invasion analysis. Transwell chambers were coated with Matrigel, which was used to simulate ECM. It was demonstrated that Tca8113 cells are able to penetrate the Matrigel into the lower chamber in the control group. At increasing concentrations of IBC, Tca8113 cells gradually lost the ability to penetrate the Matrigel. These results indicated that IBC inhibited the migratory and invasive ability of TSCC cells in a concentration-dependent manner. In order to invade and spread to the surrounding normal tissue, 
tumor cells secrete MMPs, a group of zinc-dependent endopeptidases that are able to degrade almost all extracellular matrix components (23). Aparna et al (24) identified that the high expression level of MMP-2 and MMP-9 proteins was a risk factor of local recurrence of patients with TSCC. Furthermore, MMP-9 was also identified to be associated with distant organ metastasis and survival of cancer patients. MMPs, therefore, were identified as key proteins of cancer cell invasion and metastasis. The increased expression level of MMP-2 and MMP-9 proteins represents to a certain extent the increased invasion of tongue cancer cells. In the present study, western blot analysis indicated that IBC significantly decreased the expression of MMP-2 and MMP-9 proteins in the cytoplasm of Tca8113 cells. It was suggested that IBC inhibited the invasion of Tca8113 cells by downregulating MMP-2 and MMP-9 protein expression. Previous studies have demonstrated that abnormal activation of ERK/p-ERK is able to activate a series of cytoplasmic proteins to promote the invasion and metastasis of cancer cells $(25,26)$. In addition, the Akt signaling pathway also served a role in the expression of MMP-2 and MMP-9 proteins (27). The results of the present study indicated that IBC may significantly decrease p-Akt and p-ERK protein expression in Tca8113 cells. Therefore, the inhibition of abnormal activation of the Akt and ERK signaling pathways may be one of the mechanisms of IBC inhibiting the invasiveness of Tca8113 cells.

The results of the present study confirmed that IBC inhibited Tca8113 cell proliferation, induced Tca8113 apoptosis, and exhibited an antitumor effect in a concentration- and time-dependent manner. IBC inhibited the migration and invasion of Tca8113 cells by downregulating MMP-2 and MMP-9 protein expression. Furthermore, the Akt and ERK signaling pathways may be one of the mechanisms by which IBC inhibits the invasiveness of tongue cancer cells. The present study indicated that IBC may downregulate the apoptosis-related protein Bcl-2, upregulate the expression of Bax protein, and dephosphorylate Akt and ERK proteins, in a concentration- and time-dependent manner, which may be one of the mechanisms of promoting tumor cell apoptosis. In addition, the decrease in Bcl-2 protein expression suggests that IBC may be used as a radiotherapy and chemotherapy sensitizer of TSCC in a clinical setting. The results of the present study provide an experimental basis for the clinical application of IBC in the treatment of tongue cancer and other malignancies.

\section{Acknowledgements}

The present study was supported by The First Affiliated Hospital of Liaoning Medical University (Jinzhou, China). The authors would like to thank Professor Zhi-tu Zhu for valuable support.

\section{References}

1. Clump DA, Bauman JE and Ferris RL: Cancer of the oropharynx Surg Oncol Clin N Am 24: 509-520, 2015.

2. Guo XH, Wang JY, Gao Y, Gao M, Yu GY, Xiang RL, Li L, Yang NY, Cong X, Xu XY, et al: Decreased adiponectin level is associated with aggressive phenotype of tongue squamous cell carcinoma. Cancer Sci 104: 206-213, 2013.

3. Dzebo S, Mahmutovic J and Erkocevic H: Quality of life of patients with oral cavity cancer. Mater Sociomed 29: 30-34, 2017
4. Chan KK, Glenny AM, Weldon JC, Furness S, Worthington HV and Wakeford $\mathrm{H}$ : Interventions for the treatment of oral and oropharyngeal cancers: Targeted therapy and immunotherapy. Cochrane Database Syst Rev 1: CD010341, 2015.

5. Dashtdar M, Dashtdar MR, Dashtdar B, Kardi K and Shirazi MK: The concept of wind in traditional chinese medicine. J Pharmacopuncture 19: 293-302, 2016.

6. Jing H, Zhou X, Dong X, Cao J, Zhu H, Lou J, Hu Y, He Q and Yang B: Abrogation of Akt signaling by Isobavachalcone contributes to its anti-proliferative effects towards human cancer cells. Cancer Lett 294: 167-177, 2010.

7. Nishimura R, Tabata K, Arakawa M, Ito Y, Kimura Y, Akihisa T, Nagai H, Sakuma A, Kohno H and Suzuki T: Isobavachalcone, a chalcone constituent of Angelica keiskei, induces apoptosis in neuroblastoma. Biol Pharm Bull 30: 1878-1883, 2007.

8. Szliszka E, Jaworska D, Ksek M, Czuba ZP and Król W: Targeting death receptor TRAIL-R2 by chalcones for TRAIL-induced apoptosis in cancer cells. Int J Mol Sci 13: 15343-15359, 2012.

9. Szliszka E, Czuba ZP, Mazur B, Sedek L, Paradysz A and Krol W: Chalcones enhance TRAIL-induced apoptosis in prostate cancer cells. Int J Mol Sci 11: 1-13, 2009.

10. Dzoyem JP, Hamamoto H, Ngameni B, Ngadjui BT and Sekimizu K: Antimicrobial action mechanism of flavonoids from Dorstenia species. Drug Discov Ther 7: 66-72, 2013.

11. Jin X, Zhu Z and Shi Y: Metastasis mechanism and gene/protein expression in gastric cancer with distant organs metastasis. Bull Cancer 101: E1-E12, 2014.

12. Akihisa T, Tokuda H, Hasegawa D, Ukiya M, Kimura Y, Enjo F, Suzuki T and Nishino H: Chalcones and other compounds from the exudates of Angelica keiskei and their cancer chemopreventive effects. J Nat Prod 69: 38-42, 2006.

13. Bhalla VX, Nayak UR and Dev S: Some new fiavonoids from Psoralea corylifolia. Tetrahedron Lett 20: 2401-2406, 1968.

14. Nowakowska Z: A review of anti-infective and anti-inflammatory chalcones. Eur J Med Chem 42: 125-137, 2007.

15. Green DR and Reed JC: Mitochondria and apoptosis. Science 281: 1309-1312, 1998.

16. Scott N, Hale A, Deakin M,Hand P, Adab FA, Hall C, Williams GT and Elder JB: A histopathological assessment of the response of rectal adenocarcinoma to combination chemo-radiotherapy: Relationship to apoptotic activity, p53 and bcl-2 expression. Eur J Surg Oncol 24: 169-173, 1998.

17. Zhang B, Liu M, Tang HK, Ma HB, Wang C, Chen X and Huang HZ: The expression and significance of MRP1, LRP, TOPOII $\beta$, and BCL2 in tongue squamous cell carcinoma. J Oral Pathol Med 41: 141-148, 2012.

18. Meterissian SH, Kontogiannea M, Po J, Jensen G and Ferdinand B: Apoptosis induced in human colorectal carcinoma by anti-Fas antibody. Ann Surg Oncol 4: 169-175, 1997.

19. Wang Y, Jiang XY, Liu L and Jiang HQ: Phosphatidylinositol 3-kinase/Akt pathway regulates hepatic stellate cell apoptosis. World J Gastroenterol 14: 5186-5191, 2008.

20. Chong ZZ and Maises K: Targeting WNT, protein kinase B, and mitochondrial membrane integrity to foster cellular survival in the nervous system. Histol Histopathol 19: 495-504, 2004.

21. Aranda F, Vacchelli E, Eggermont A, Galon J, Fridman WH, Zitvogel L, Kroemer G and Galluzzi L: Trial Watch: Immunostimulatory monoclonal antibodies in cancer therapy. Oncoimmunology 3: e27297, 2014.

22. Jin X and Shi YI: Isobavachalcone induces the apoptosis of gastric cancer cells via inhibition of the Akt and Erk pathways. Exp Ther Med 11: 403-408, 2016.

23. Rao JS: Molecular mechanisms of glioma invasiveness: The role of proteases. Nat Rev Cancer 3: 489-501, 2003.

24. Aparna M, Rao L, Kunhikatta V and Radhakrishnan R: The role of MMP-2 and MMP-9 as prognostic markers in the early stages of tongue squamous cell carcinoma. J Oral Pathol Med 44: 345-352, 2015.

25. Yin B, Liu Z, Wang Y, Wang X, Liu W, Yu P, Duan X, Liu C, Chen Y, Zhang Y, et al: RON and c-Met facilitate metastasis through the ERK signaling pathway in prostate cancer cells. Oncol Rep 37: 3209-3218, 2017.

26. Wang D, Wang D, Wang N, Long $Z$ and Ren $X$ : Long non-coding RNA BANCR promotes endometrial cancer cell proliferation and invasion by regulating MMP2 and MMP1 via ERK/MAPK signaling pathway. Cell Physiol Biochem 40: 644-656, 2016.

27. Cheng CY, Hsieh HL, Hsiao LD and Yang CM: $\mathrm{PI} 3-\mathrm{K} / \mathrm{Akt} / \mathrm{JNK} / \mathrm{NF}-\kappa \mathrm{B}$ is essential for MMP-9 expression and outgrowth in human limbal epithelial cells on intact amniotic membrane. Stem Cell Res 9: 9-23, 2012. 